\title{
DELIMITAÇÃO DO TOPO DE AQÜÍFERO NA REGIÃO DE PORTO SEGURO - BAHIA ATRAVÉS DA INVERSÃO DE SONDAGENS ELÉTRICAS VERTICAIS
}

\author{
Alexandre C. Monteiro \& Milton J. Porsani
}

Received April 22, 2000 / Accepted November 12, 2002

\begin{abstract}
O presente trabalho compreendeu estudos geofísicos conduzidos com o método da eletrorresistividade na região de Porto Seguro (BA), que tiveram o objetivo de localizar o aqüífero mais promissor para a exploração de água subterrânea naquela região. Os trabalhos recobriram uma área de aproximadamente $60 \mathrm{~km}^{2}$ situada imediatamente ao norte de Porto Seguro. A área pesquisada insere-se num contexto geológico de ambiente sedimentar Terciário (Formação Barreiras) e Quaternário (cordões litorâneos). Foram realizadas 60 sondagens elétricas verticais (SEVs) utilizando o arranjo Schlumberger. Destas, 14 foram realizadas para reconhecimento regional; 36 localizaram-se dentro da área de interesse e 10 distribuiram-se ao longo de um perfil regional ligando as cidades de Eunápolis e Porto Seguro. Estas últimas foram realizadas com o objetivo de delinear o topo do embasamento cristalino entre aquelas cidades. Para a interpretação quantitativa das SEVs foi desenvolvido um programa Fortran baseado no método de inversão que utiliza a técnica da inversão linearizada com reponderação múltipla dos desvios (ILRMD). Este método foi testado na inversão de SEVs sintéticas e em seguida aplicado para inversão das SEVs obtidas nos trabalhos de campo. Os resultados demonstraram que o método de ILRMD é mais robusto e eficaz comparado ao método de inversão linearizada governado pela norma $\mathrm{L}^{2}$ ou $\mathrm{L}^{1}$. Para auxiliar a interpretação foram utilizados dados disponíveis de perfis litológicos de poços perfurados na região. Utilizando-se as estimativas de espessura e resistividades de subsuperfície, obtidas através das inversões das SEVs, foram construídas seções geoelétricas. A interpretaçcão destas seções permitiu determinar o aqüífero de interesse para a exploração de água subterrânea naquela região. Este é formado predominantemente por arenito saturado com água doce e ocupa a porção centro-norte da área com o topo na profundidade a cerca de $80 \mathrm{~m}$ e espessura não inferior a $70 \mathrm{~m}$. Tal aqüífero representa a melhor opção para a captação de água subterrânea no município de Porto Seguro.
\end{abstract}

Palavras-chave: Eletrorresistividade; Inversão de SEV; Delineação do aqüífero; Porto-Seguro.

\begin{abstract}
DEFINITION OF THE AQUIFER TOP IN THE REGION OF PORTO SEGURO-BAHIA FROM THE INVERSION OF VERTICAL ELECTRICAL SOUNDING DATA - This paper comprises geophysical studies carried out with the resistivity method in the Porto Seguro region, in the state of Bahia (Brazil). The objective was to find the main aquifer of that region. The work was performed on a area of approximately $60 \mathrm{~km}^{2}$ located north of the Porto Seguro city. The studied area has sedimentary units with Tertiary (Formação Barreiras) and Quaternary (coastal shore) ages. Sixty vertical electrical soundings (VES) were performed using the Schlumberger array: 14 for regional reconnaissance; 36 for detailed study and 10 in a profile between the cities of Eunápolis and Porto Seguro. The VES in the regional profile were carried out to define the basement topography between these cities. A Fortran program was written for the quantitative interpretation of the VES, based on the method that uses the linearized inversion with multiple weight for the deviations (LIMWD). This proposition was tested on synthetic VES data and then it was used with field data. The results showed that this method is more stable and powerful than the traditional linearized inversion method which uses $L^{1}$ and $L^{2}$ fixed norms. The available information about well drilling data of the region was used to aid the interpretation. Geoelectrical sections were drawn from the inverted results of the thickness and resistivity of the layers. The interpretation
\end{abstract}


of these sections provided the definition of the main aquifer for ground water exploitation in this region. This aquifer is confined to saturated sandstone layer in the mid-north portion of the area at about $80 \mathrm{~m}$ deep and an average thickness of $70 \mathrm{~m}$. This aquifer represents the best option for ground water exploitation to supply the city of Porto Seguro.

Key words: Electro-resistivity survey; VES inversion; Aquifer mapping; Porto-Seguro city.

\author{
${ }^{1}$ CPRM, Divisão de Geofísica, Rio de Janeiro, RJ, Brazil \\ E-mail: acm@cristal.cprm.gov.br \\ ${ }^{2}$ Centro de Pesquisa em Geologia e Geofísica \\ Instituto de Geociências, Universidade Federal da Bahia \\ Campus Universitário da Federação, Salvador, Bahia, Brazil \\ E-mail: porsani@cpgg.ufba.br
}

\section{INTRODUÇÃO}

A região de Porto Seguro na Bahia recebe um enorme fluxo turístico ao longo do ano. A maior parte da água consumida advém de poços perfurados no principal aquíf́ero associado à Formação Barreiras, que é a principal unidade estratigráfica da área. Com o objetivo de delinear o topo daquele aquífero trabalhamos com o método geofísico de eletrorresistividade, tradicionalmente utilizado para a pesquisa de água subterrânea.

Foram realizadas três campanhas de campo com a execução de 60 sondagens elétricas verticais (SEVs). A primeira etapa compreendeu a realização de um reconhecimento geofísico regional. Na segunda etapa procedeu-se ao adensamento da malha de sondagens, priorizando-se a área imediatamente ao norte da cidade de Porto Seguro situada entre as coordenadas $-39^{\circ} 08^{\prime},-16^{\circ} 27^{\prime}$ e $-39^{\circ} 00^{\prime},-16^{\circ} 22^{\prime}$ (IBGE, 1987). Sua escolha foi determinada pela proximidade com a cidade de Porto Seguro, que é o maior centro populacional da região e que vem sendo submetida a intenso crescimento populacional (condomínios, hotéis, favelas) desde o início da década passada. Trata-se de uma região com problemas de abastecimento de água, visto que os mananciais superficiais estão sendo gradativamente assoreados e/ou poluídos devido a uma ocupação desordenada nas proximidades da cidade. Ao todo, dentro da área selecionada, foram realizadas $36 \mathrm{SEV}$ s além de outras 10 SEVs, dispostas ao longo de um perfil regional interligando as cidades de Eunápolis (situa-se a $65 \mathrm{~km}$ a oeste) e Porto Seguro, e realizadas com o objetivo de se delinear o topo do embasamento cristalino entre aquelas cidades.

\section{GEOLOGIA DA ÁREA}

Conforme ilustra a Fig. 1, a geologia da área de estudo está totalmente inserida na Formação Barreiras e nos cordões litorâneos de idade Quaternária (Bittencourt et al., 1979, Barbosa et al., 1996, IBGE, 1987). A Formação Barreiras é constituida de sedimentos continentais costeiros de idade Terciária (Mioceno-Plioceno), que formam extensos tabuleiros, freqüientemente cortados por falésias junto a linha de costa. Os principais constituintes litológicos na área são arenitos maturos que mostram cores variadas, comumente com estratificação plano-paralela e secundariamente estratificação cruzada. A fração pelítica é representada por siltitos e folhelhos intercalados aos arenitos em camadas de espessuras variando de alguns centímetros a dezenas de metros. A espessura do pacote sedimentar na área, obtida de perfis litológicos de poços perfurados é maior do que $150 \mathrm{~m}$. Os depósitos Quaternários são representados principalmente por sedimentos flúvio-marinhos e fluviais. Os primeiros dispõem-se ao longo de toda a costa e principalmente na desenbocadura do rio Buranhém, ao sul da área. Os cordões litorâneos ocorrem entre a linha de praia atual e a base das falésias da Formação Barreiras, correspondendo a praias antigas, ora apresentando-se paralelos, ora oblíquos às praias atuais. São depósitos arenosos contendo minerais pesados. Os aluviões são caracterizados por material arenoso e areno-argiloso, no leito e nas planícies de inundação e terraços dos principais rios. 


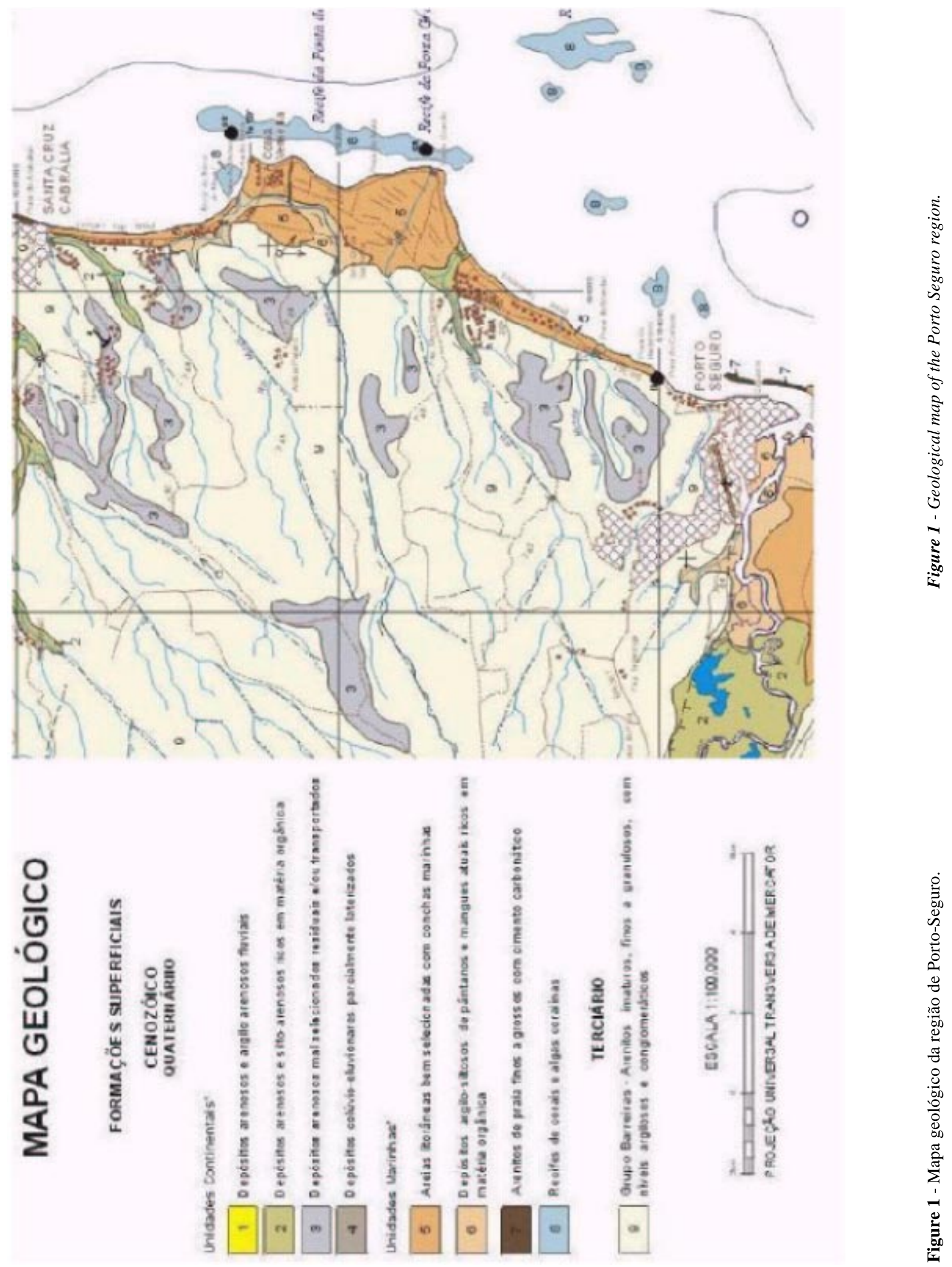


CARACTERÍSTICAS DAS SEVS NA ÁREA DE ESTUDO

Na Fig. 2 estão apresentadas todas as curvas de SEVs realizadas na área de estudo. Énítida a tendência que a maioria possui de se comportar como curvas do tipo Q (Tab. 1) (Keller \& Frischknecht, 1966) As SEVs que possuem esta tendência situam-se sobre a Formação Barreiras. Sob influência da camada superficial, que pode variar de areia até argila as SEVs iniciam-se com resistividade aparente entre $100 \Omega \mathrm{m} \mathrm{a}$
$10.000 \Omega \mathrm{m}$. Passada o trecho inicial da curva, os valores de resistividade aparente estabilizam em torno de $600 \Omega \mathrm{m}$, e perduram até a abertura $A B / 2$ atingir 100 m aproximadamente. A partir daí, a resistividade aparente começa a decrescer de forma marcante até $\mathrm{AB} / 2$ atingir $400 \mathrm{~m}$, quando então a curva muda a sua tendência de queda e começa a subir sob a influência do embasamento cristalino resistivo.

As SEVs que foram realizadas nas praias e zona litorânea (abaixo das falésias da Formação Barreiras) obedecem, de maneira geral, ao padrão típico de

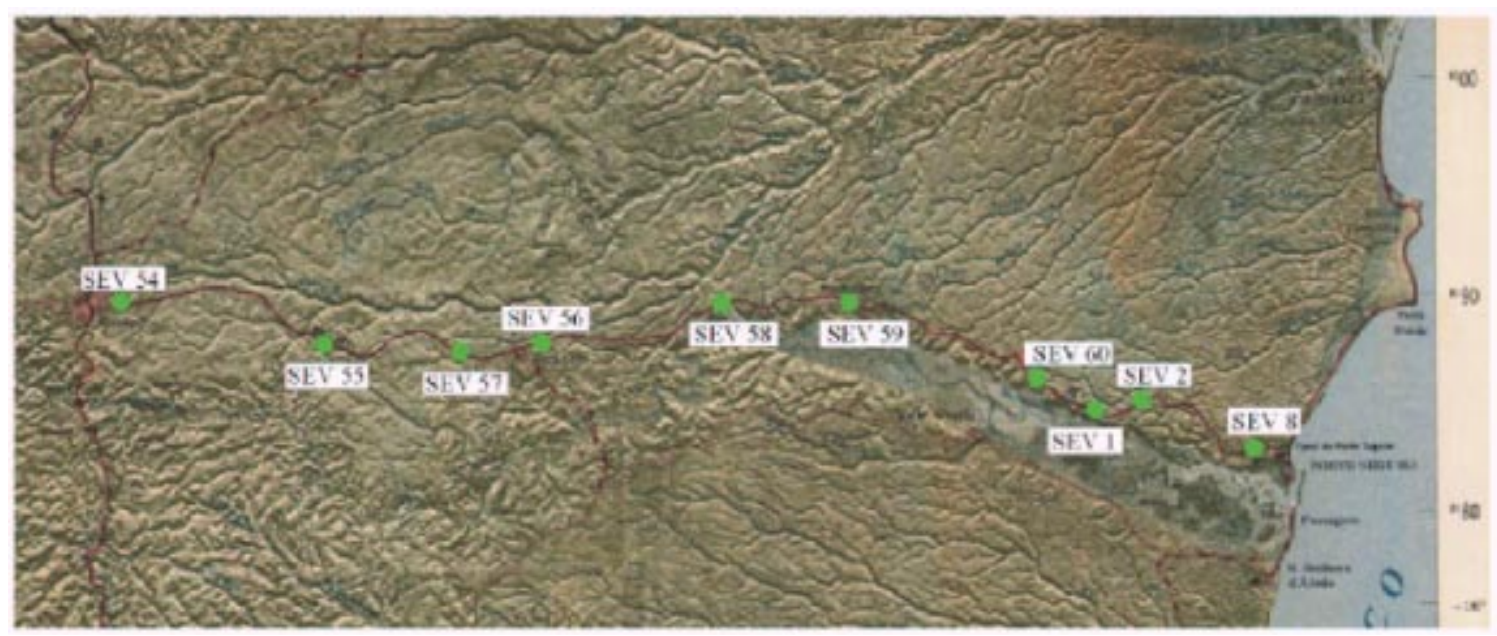

Figura 4 - Imagem de radar com a localização das SEVs do perfil Eunápolis - Porto Seguro.

Figure 4 - Radar image showing the location of VES along the profile between the cities of Eunápolis and Porto Seguro.

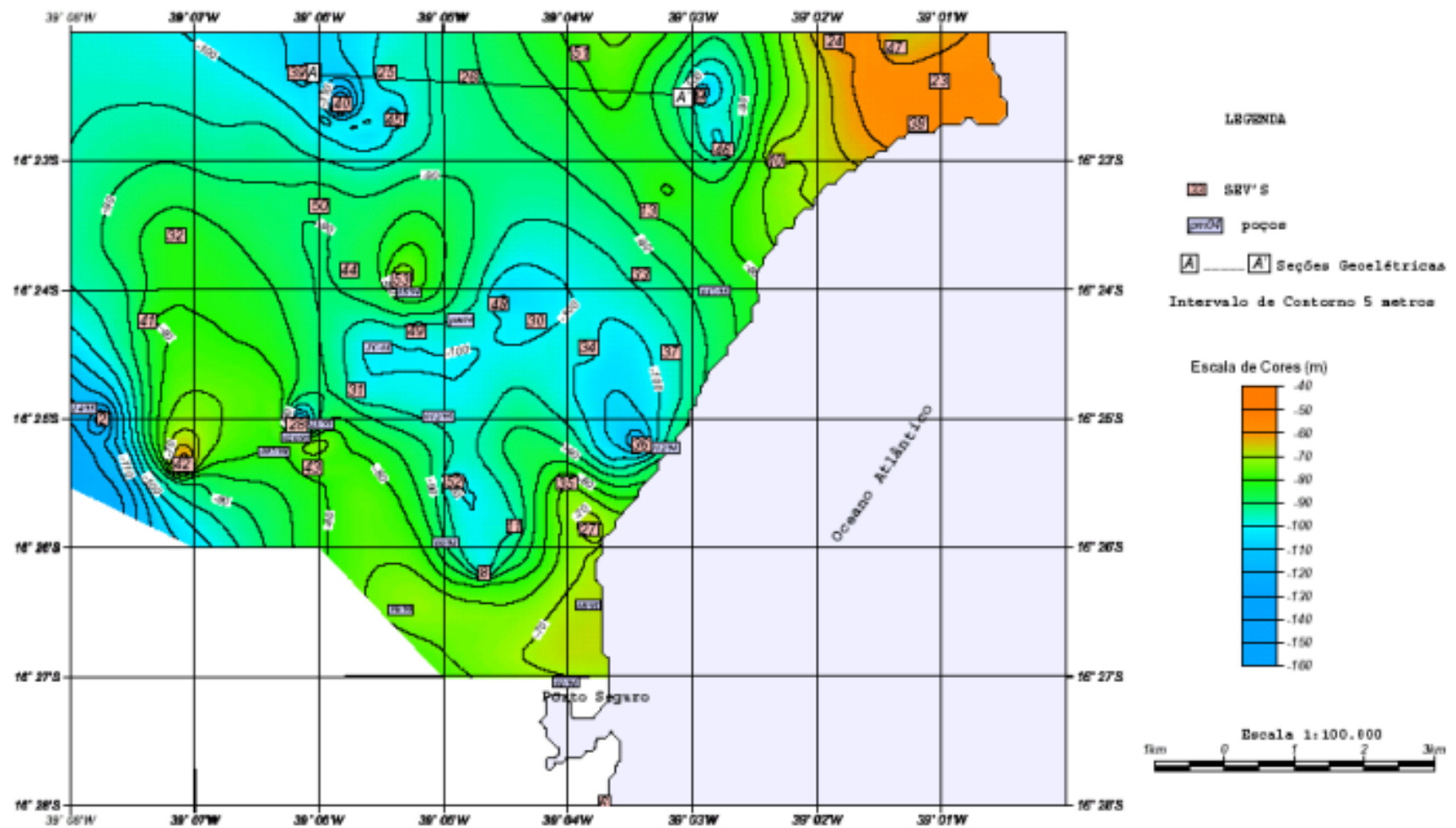

Figura 9 - Mapa do topo do aqüífero profundo.

Figure 9 - Map of the top of the deep aquifer. 
curvas do tipo $\mathrm{H}$ (Tab. 1) como pode ser visto nas 4 curvas inferiores da Fig. 2. Tais curvas fogem da tendência geral descrita e formam uma segunda família de curvas. O comportamento distinto destas curvas é coerente com a geologia local formada por sedimentos de idade Quaternária e a presença de água salobra a cerca de $8 \mathrm{~m}$ de profundidade (informação verbal obtida na área) que é responsável pelos valores baixos de resistividade aparente no intervalo $10 \mathrm{~m}<\mathrm{AB} / 2<$ $100 \mathrm{~m}$.

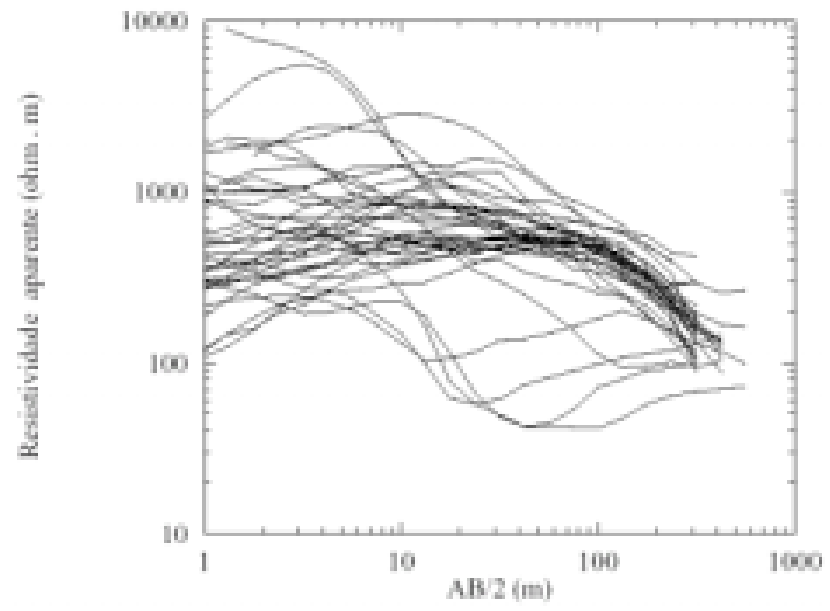

Figura 2 - Curvas das SEVs executadas na área de estudo exibidas em grupo.

Figure 2 - Curves of the VES carried out in the studied area displayed as a group.

\section{INVERSÃO LINEARIZADA COM REPONDERAÇÃO MÚLTIPLA DOS DESVIOS}

A solução do problema inverso consiste em encontrar o modelo $\tilde{\mathbf{m}}$ que, através da equação teórica utilizada para cálculo das resistividades aparentes, melhor descreva as resistividades aparentes

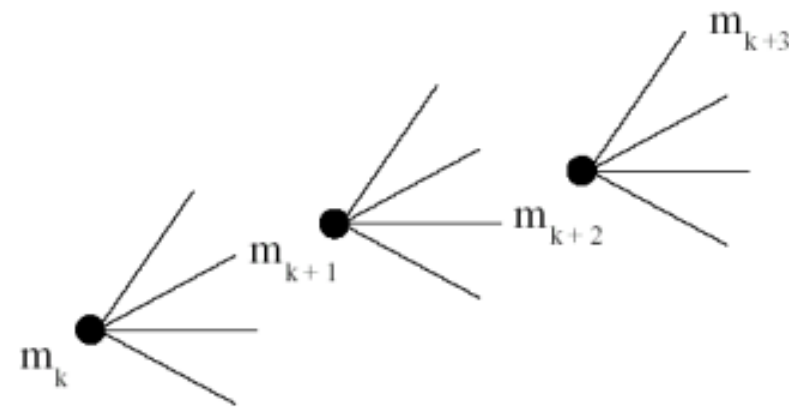

Figura 3 - Representação esquemática da forma como o método ILRMD explora o espaço dos modelos. A cada iteração vários modelos candidatos são visitados a partir do modelo corrente. Tão logo seja encontrado um melhor modelo, este substitui o modelo corrente. Caso não seja encontrado nenhum modelo melhor que o modelo corrente, ainda assim o modelo corrente dá lugar ao melhor modelo encontrado.

Figure 3 - Schematic representation of the way the ILRMD method explores the model space. At each iteration many candidate models are visited as of the current model. As soon as a better model is found it substitutes the current model. If no better models are found, even then the current model gives room to the best model found.

\section{Seção Geoelétrica Eunápolis - Porto Seguro}

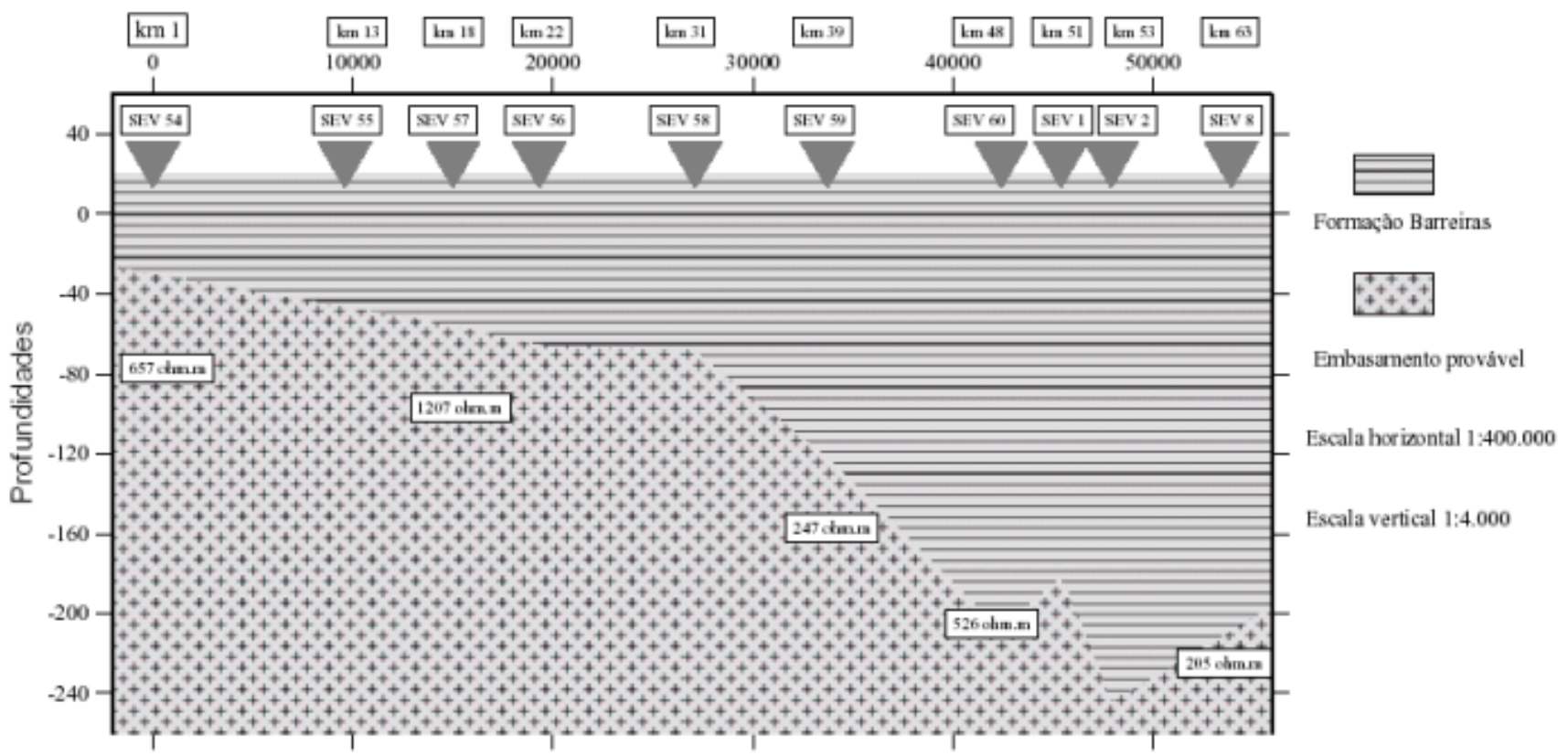

Figura 5 - Seção geolétrica mostrando a topografia do embasamento entre as cidades de Eunápolis e Porto Seguro.

Figure 5 - Geoelectrical section showing the topography of the basement between the cities of Eunápolis and Porto Seguro. 


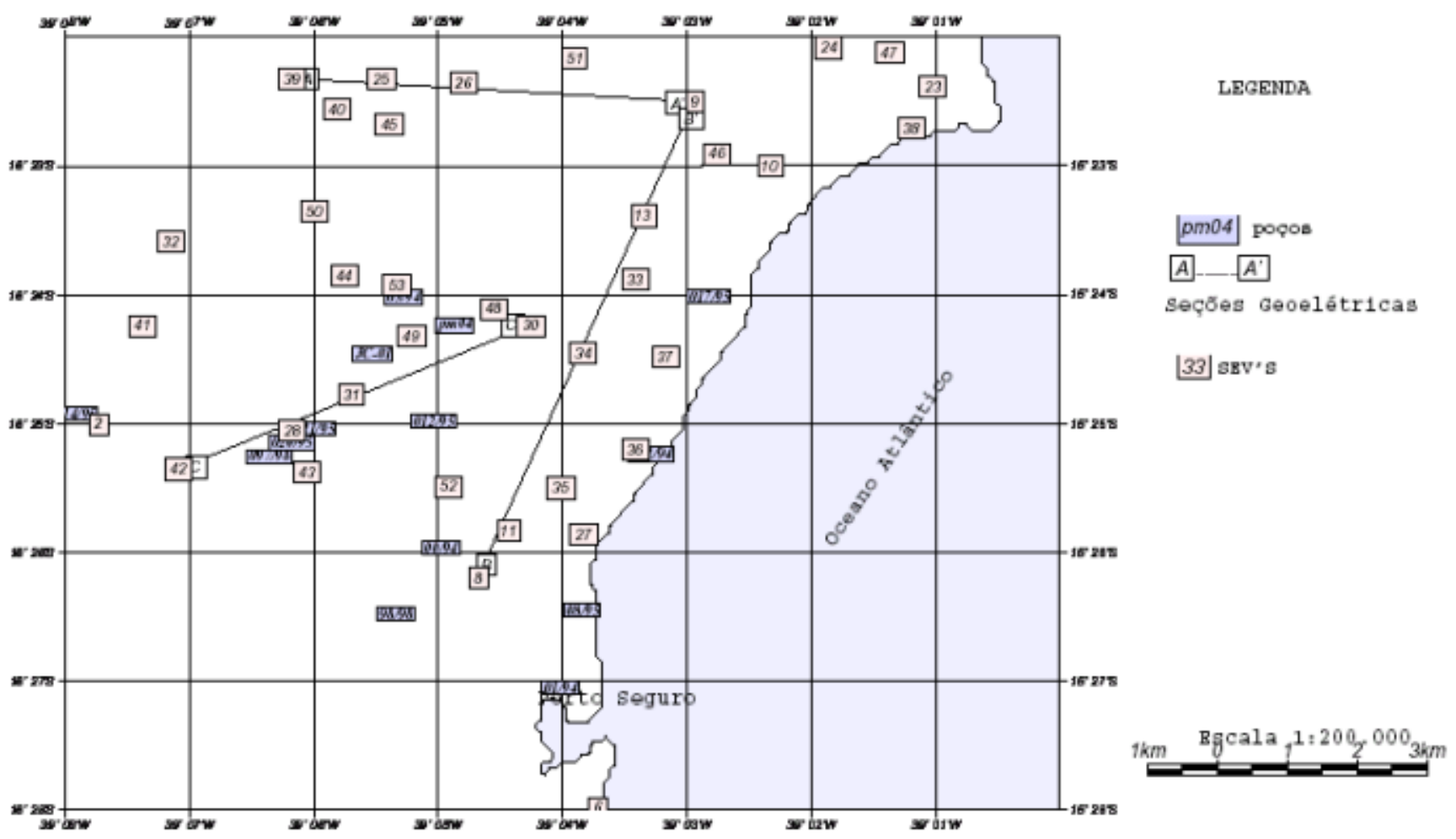

Figura 6 - Mapa de localização das SEVs realizadas na região de Porto-Seguro.

Figure 6 - Map of the VES carried out in the Porto Seguro region.
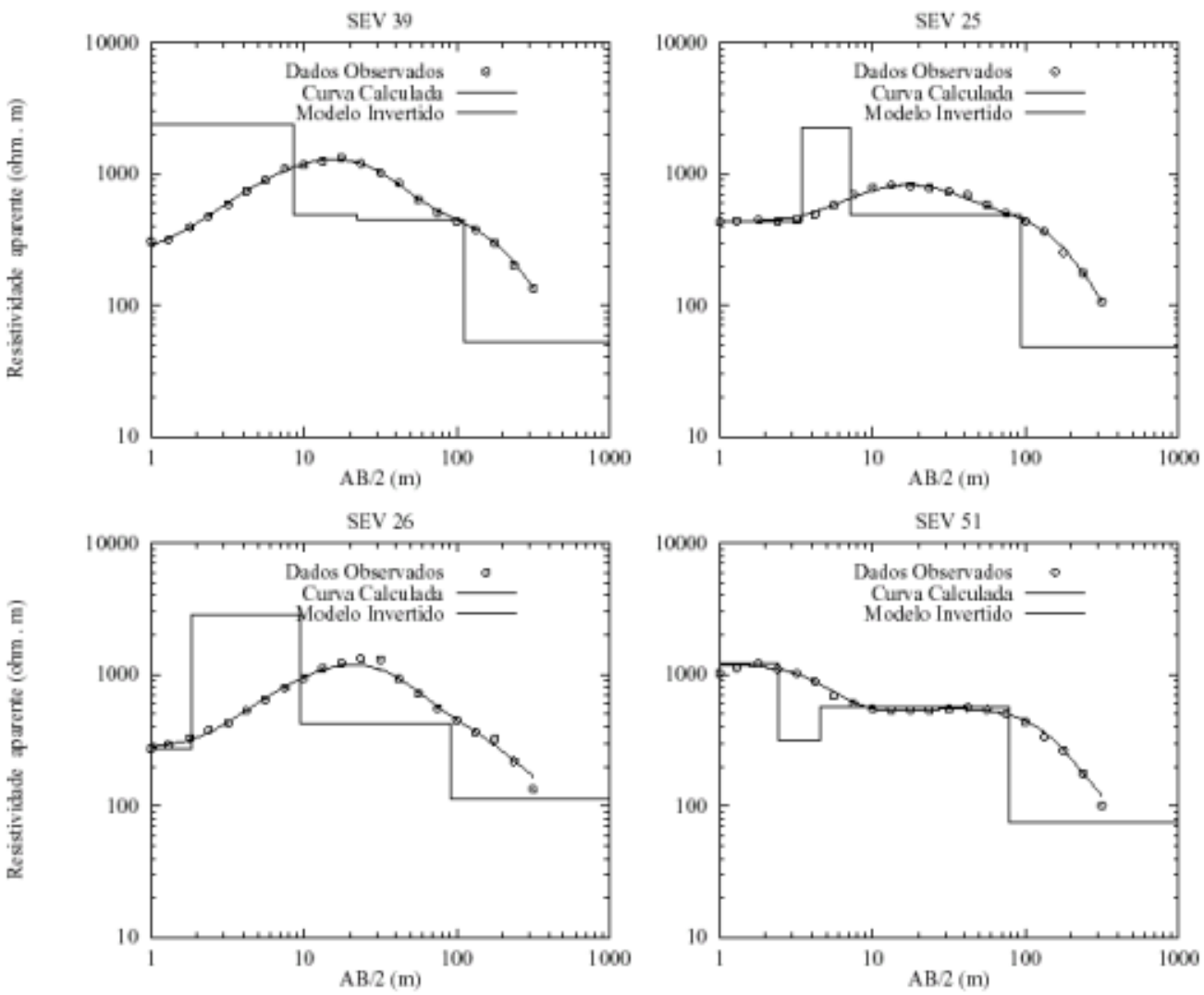

Figura 7 - Resultados da inversão de SEVs da seção A - A' com o método ILRMD mostrando o topo do aqüífero na profundidade aproximada de $100 \mathrm{~m}$.

Figure 7 - Results of the VES inversion along section A - A' using the LIMWD method showing the top of the aquifer located at approximately $100 \mathrm{~m}$ deep. 


\begin{tabular}{|c||c|}
\hline \multicolumn{2}{|c|}{ Modelos de 3 camadas } \\
\hline tipo $\mathrm{A}$ & $\rho_{1}<\rho_{2}<\rho_{3}$ \\
\hline tipo $\mathrm{H}$ & $\rho_{1}>\rho_{2}<\rho_{3}$ \\
\hline tipo $\mathrm{K}$ & $\rho_{1}<\rho_{2}>\rho_{3}$ \\
\hline tipo Q & $\rho_{1}>\rho_{2}>\rho_{3}$ \\
\hline
\end{tabular}

Tabela 1 - Valores de resistividades para modelos de 3 camadas correspondentes aos 4 tipos padrões de curvas de SEVs.

Table 1 - Resistivity values for 3 layers model corresponding to the 4 standard types of VES.

observadas (Rijo et al., 1977; Lines \& Treitel, 1984; Carrasquilla \& Rijo, 1991; Ferreira \& Porsani, 1977; Marinho, 1997; Monteiro \& Porsani, 1999; Monteiro, 1999). A seguir apresentamos o método utilizado na interpretação quantitativa das SEVs desenvolvido com base no procedimento iterativo proposto por Porsani et al. (2001).

Seja d(x) o valor de resistividade aparente de uma SEV, observado na separação $\mathrm{x}=\mathrm{AB} / 2$ e seja $\rho_{\mathrm{a}}(\mathbf{m}, \mathrm{x})$ a função não linear utilizada para cálculo dos valores teóricos de resistividade aparente (Gosh, 1971a, 1971b; Johansen, 1977; Koefoed,1979). O vetor m representa as resistividades, $\rho_{j}$, e espessuras, $h_{j}$, do modelo de $\mathrm{N}$ camadas, $\mathbf{m}=\left(\rho_{1}, \ldots, \rho_{\mathrm{N}}, \mathrm{h}_{1}, \ldots \mathrm{h}_{\mathrm{N}-1}\right)^{\mathrm{T}}$.

Os desvios entre as resistividades aparentes observadas e calculadas podem ser colocados em termos de uma dependência exponencial como segue,

$$
\begin{gathered}
f(\mathbf{m}, p, x)=\left\{\left[\Delta d^{2}(\mathbf{m}, x)\right]^{1 / 2}\right\}^{p}= \\
\left\{\left[\left(d(x)-\rho_{a}(\mathbf{m}, x)\right)^{2}\right]^{1 / 2}\right\}^{p} .
\end{gathered}
$$

Expandindo a equação (1) em série de Taylor em torno do modelo $\mathbf{m}_{\mathrm{k}}$, tomando a aproximação de primeira ordem e avaliando-a em $\mathrm{x}_{\mathrm{i}}, \mathrm{i}=1, \ldots, \mathrm{M}$, obtém-se o sistema linear de equações representado a seguir,

$$
\begin{gathered}
\mathrm{f}(\mathrm{m}, p, \mathrm{x}) \approx \tilde{\mathrm{f}}(\mathrm{m}, p, \mathrm{x})= \\
\mathrm{f}_{p k}-\mathrm{W}_{p k} \mathrm{G}_{k} \Delta \mathrm{m},
\end{gathered}
$$

onde $\mathrm{x}=\left(\mathrm{X}_{1}, \ldots, \quad \mathrm{X}_{\mathrm{M}}\right)^{\mathrm{T}}$ representa o vetor da variável independente associada às posições das medidas; $\mathrm{G}_{\mathrm{k}}$ representa a matriz sensibilidade, na qual cada coluna corresponde à derivada de $\rho_{\mathrm{a}}(\mathbf{m}, \mathrm{x})$, tomada com relação a cada parâmetro do modelo e avaliada nas posições $x_{i}$; o vetor $f_{p k}=f\left(m_{k}, p, x\right)$ representa os desvios associados ao modelo $\mathbf{m}_{\mathrm{k}}$, calculados através da Eq. (1); $\Delta \mathbf{m}=\mathbf{m}-\mathbf{m}_{\mathrm{k}}$ representa a correção a ser dada no modelo corrente $\mathbf{m}_{\mathrm{k}}$ e $\mathrm{W}_{\mathrm{pk}}$ representa uma matriz diagonal de pesos, com elementos ${ }^{\mathrm{k}} \mathrm{W}_{\mathrm{ii}}$ dados por,

$$
{ }^{k} w_{i i}=\frac{p}{2}\left[\Delta d^{2}\left(\mathrm{~m}_{k}, x_{i}\right)\right]^{p / 4-1} \Delta d\left(\mathrm{~m}_{k}, x_{i}\right),
$$

e os elementos do vetor $f_{p k}$ são dados por,

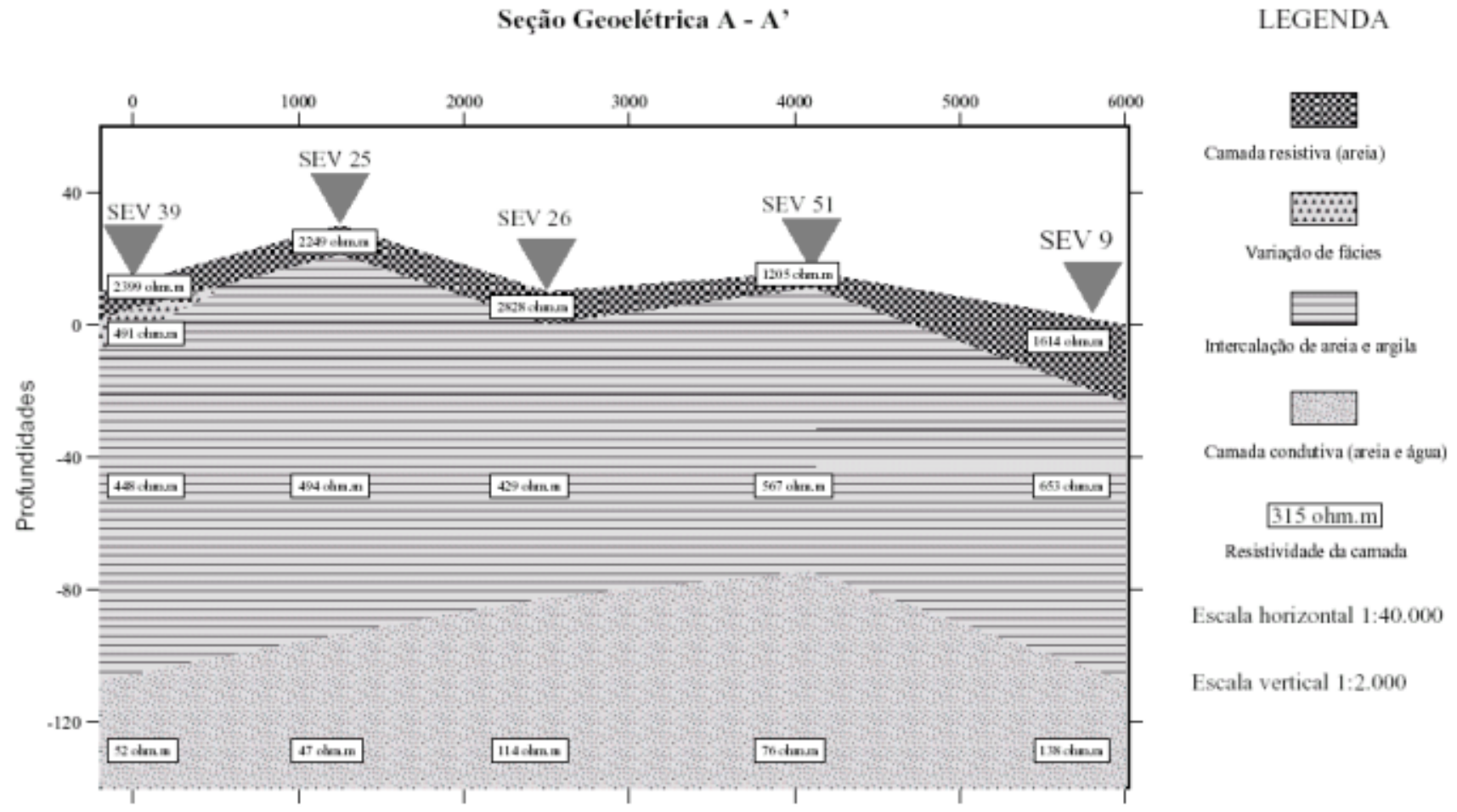

Figura 8 - Seção geoelétrica no norte da área ( A -A').

Figure 8 - Geoelectrical section in the northern part of the area ( $\left.A-A^{\prime}\right)$. 


$$
f_{i}=\left[\Delta d^{2}\left(\mathrm{~m}_{k}, x_{i}\right)\right]^{p / 4} .
$$

Observa-se na Eq. (2) que $\tilde{f}(\mathbf{m k}, \mathbf{p}, \mathbf{x})$ é uma função linear em $\Delta \mathbf{m}$. Pode-se, para qualquer valor de p, obter $\Delta \mathbf{m}$ associado ao mínimo da forma quadrática $\Sigma \tilde{f}^{2}\left(\mathbf{m}, \mathrm{p}, \mathrm{x}_{\mathrm{j}}\right)$. Desta forma obtém-se a expressão utilizada para atualização do modelo corrente,

$$
\mathrm{m}_{k+1}=\mathrm{m}_{k}+\left(\mathrm{G}_{k}^{T} \mathrm{~W}_{p k}^{2} \mathrm{G}_{k}\right)^{-1} \mathrm{G}_{k}^{T} \mathrm{~W}_{p k} \mathrm{f}_{p k} .
$$

O método de inversão linearizada (IL), conduzido através da Eq. (5), pode ser visto como um procedimento iterativo no qual a linha i da matriz sensibilidade é ponderada pelo fator ${ }^{\mathrm{k}} \mathrm{W}_{\mathrm{ii}}$ que depende do desvio entre os valores de resistividades aparentes observadas e aqueles calculados a partir do modelo corrente $\mathbf{m}_{\mathrm{k}}$.

Uma equação semelhante foi derivada por Scales \& Gersztenkorn (1988) para o método de IL com norma Lp. Outro algoritmo proposto por Egbert \& Booker (1986) atribui peso pequeno aos pontos com ajuste discrepantes. Para $\mathrm{p}=2$ a Eq. (5) resulta igual à expressão normalmente utilizada no método de IL com base na norma $\mathrm{L}^{2}$,

$$
\mathrm{m}_{k+1}=\mathrm{m}_{k}+\left(\mathrm{G}_{k}^{T} \mathrm{G}_{k}\right)^{-1} \mathrm{G}_{k}^{T} \Delta d\left(\mathrm{~m}_{k}, \mathrm{x}\right)
$$

Deixando $\mathrm{p}=1$ obtém-se a equação para IL com base na norma $\mathrm{L}^{1}$. Se desejarmos restringir o uso da equação de forma a satisfazer a definição matemática de norma $L^{p}$ é suficiente escolher $p \geq 1$. Também, de maneira análoga à norma $\mathrm{L}^{2}$ pode-se utilizar os procedimentos de "regularização" (Levenberg, 1944; Foster, 1961; e Marquardt, 1963 in Pelton et al., 1978) para assegurar estabilidade numérica da solução da Eq. (5).

A Eq. (5) flexibiliza o uso do método de IL. Ela nos dá a possibilidade de ponderarmos exponencialmente os desvios com um parâmetro $\mathrm{p}$ que pode ser mantido fixo ou não durante as iterações. Nota-se ainda que, para um dado modelo corrente $\mathbf{m}_{\mathrm{k}}$, e sua correspondente matriz sensibilidade $\mathrm{G}_{\mathrm{k}}$ a atualização do modelo corrente depende apenas do parâmetro p. Considerando que na maioria das vezes não se conhece a função distribuição dos erros nas observações não se pode arbitrar pelo uso da norma
$\mathrm{L}^{2}(\mathrm{p}=2)$ ou $\mathrm{L}^{1}(\mathrm{p}=1)$ ou um valor qualquer de $\mathrm{p}$ para governar a inversão.

Sabe-se também que na solução de problemas inversos de geofísica através do método da IL, a resolução do sistema de equações corresponde apenas a uma fração do custo computacional gasto para obtenção da matriz $\mathrm{G}_{\mathrm{k}}$. Esse fato motiva e justifica o uso da Eq. (5) empregando diferentes valores de $\mathrm{p}$ em cada iteração, conforme ilustra a Fig. (3). A esta forma de utilização da Eq. (5) denominamos de IL com reponderação múltipla dos desvios (ILRMD). Desta maneira, em cada passo k do processo iterativo, podemos pesquisar várias vezes por um melhor modelo para substituir o modelo corrente. Esta estratégia, proposta por Porsani et al. (2001), permite que se explore o espaço dos modelos de forma mais extensiva, conferindo robustez e eficácia ao método de IL.

\section{MEDIDA DA QUALIDADE DO AJUSTE}

Para avaliar a qualidade do ajuste e, por conseguinte, proceder a escolha do melhor modelo várias equações podem ser utilizadas (Sen \& Stoffa, 1995). No presente trabalho adotamos a equação definida por Porsani et al. (2000), que quantifica o desempenho de cada modelo utilizando os desvios elevados à uma determinada potência conforme ilustra a equação abaixo,

$$
\begin{gathered}
\Phi(\mathrm{m})=1- \\
\frac{2 \sum_{j=1}^{N}\left|\Delta d\left(\mathrm{~m}, x_{j}\right)\right|^{1 / 2}}{\sum_{j=1}^{N}\left|\Delta d\left(\mathrm{~m}, x_{j}\right)\right|^{1 / 2}+\sum_{j=1}^{N}\left|\Delta f\left(\mathrm{~m}, x_{j}\right)\right|^{1 / 2}}
\end{gathered}
$$

onde $\Delta f\left(m, x_{j}\right)=d\left(x_{i}\right)+\rho_{a}\left(m, x_{i}\right)$. Nota-se que, se $d(x)=\rho_{a}(\mathbf{m}, x)$, a Eq. (7) resulta igual a 1 .

No Apêndice apresentamos os passos do algoritmo de ILRMD e resultados obtidos na inversão de diferentes tipos de curvas de SEVs sintéticas.

\section{INVERSÃO DE SEVS DE CAMPO}

Os experimentos de inversão de curvas de SEVs sintéticas (Apêndice), semelhantes às obtidas na área de trabalho, permitiram definir a faixa de valores de $\mathrm{p}$ apropriada para a inversão das SEVs de campo. Para fins de comparação também foram feitas tentativas 
com os valores $\mathrm{p}=2$ (norma $\mathrm{L}^{2}$ ) e $\mathrm{p}=1$ (norma $\mathrm{L}^{1}$ ). Conforme esperado, de forma análoga à inversão das SEVs sintéticas, a IL conduzida com norma fixa se mostrou instável mesmo utilizando-se o fator de amortecimento de Marquardt-Levenberg. No método ILRMD algumas vezes a mesma instabilidade também apareceu. Nestes casos, a inversão foi estabilizada incrementando-se os elementos da diagonal da matriz dos coeficientes de um percentual $(0.1 \%)$.

\section{PERFIL REGIONAL EUNÁPOLIS-PORTO SEGURO}

Com o objetivo de se delinear o topo do embasamento cristalino no trecho Eunápolis - Porto Seguro foi realizado um perfil geofísico de caráter regional com $10 \mathrm{SEV}$ s distribuidas ao longo da BR367 (Fig. 4, na pág. 282). A primeira (SEV 54) se situa a uma distância de $1 \mathrm{~km}$ de Eunapólis e, a última, ao lado da pista do aeroporto de Porto Seguro (SEV $8)$, a cerca de $1 \mathrm{~km}$ do centro da cidade.

Como pode ser visto na seção geoelétrica representada na Fig. 5 (pág. 283), o embasamento em Eunapólis está a uma profundidade aproximada de $30 \mathrm{~m}$. Em direção a Porto Seguro, o pacote sedimentar aumenta gradualmente sua espessura até a altura da SEV 58 no $\mathrm{km} \mathrm{31.} \mathrm{A} \mathrm{partir} \mathrm{deste} \mathrm{ponto} \mathrm{o}$ pacote sedimentar aumenta de forma significativa de $60 \mathrm{~m}$ para $120 \mathrm{~m}$ na SEV 59, alcançando $200 \mathrm{~m}$ na SEV 60 e mantendo-se neste patamar nas proximidades de Porto Seguro. Este comportamento da geologia pode ser explicado por duas falhas transcorrentes mapeadas no projeto Porto Seguro Santa Cruz de Cabrália (Saadi, 2000): ambas possuem direção NE-SW situando-se a primeira junto a SEV 58 e a segunda pouco antes da SEV 60. Estas estruturas geológicas são interpretadas aqui como deslocamentos verticais associados a estas falhas transcorrentes mapeadas, portanto estes deslocamentos verticais devem ser responsáveis pelo maior espessamento do pacote sedimentar nas proximidades de Porto Seguro.

\section{PERFIL A - A'}

A localização do perfil encontra-se indicada na Fig. 6. A Fig. 7 mostra os resultados da inversão de
4 das 5 SEVs daquele perfil mostrando o topo da camada aquíf́era (arenito saturado) na profundidade de $100 \mathrm{~m}$ aproximadamente. A Fig. 8 apresenta a seção geoelétrica interpretada. A interpretação é consistente com os dados litológicos de poços disponíveis ao sul da área. O modelo geofísico/ geológico interpretado, foi discutido com o geólogo Alberto Vieira que perfurou cerca de 100 poços na região, vários dentro da área de estudo. Interpretouse uma camada superficial com menos de $1 \mathrm{~m}$ de espessura que foi omitida na figura. A seguir existem basicamente três horizontes geoelétricos de interesse. $\mathrm{O}$ primeiro com resistividade alta em torno dos $1800 \Omega \mathrm{m}$, que deve se tratar de um material arenoso, podendo atingir até os $20 \mathrm{~m}$ de espessura. O segundo possui espessura em torno de $60 \mathrm{~m}$ e foi interpretada como sendo uma alternância de areia e argila, podendo conter aqüíferos confinados ou não, bem como camadas não saturadas, uma vez que o nível piezométrico nesta região bem como em toda a área, por sobre a Formação Barreiras, encontra-se aproximadamente a $40 \mathrm{~m}$ de profundidade. A última camada geoelétrica detectada nas SEVs possui resistividade menor que as anteriores e situa-se em torno dos $80 \mathrm{~m}$ de profundidade. Esta corresponde a um espesso pacote de arenito saturado por água doce e se constitui na opção mais promissora para captação água subterrânea de boa qualidade.

\section{MAPA DO AQÜÍFERO PROFUNDO}

Foi feito então um mapa contendo o topo desta "última camada" aqui sendo interpretada como o topo do principal aquiífero desta área (Fig. 9, na pág. 284). As camadas superiores, que nas SEVs comportamse como um pacote com resistividade em torno de $500 \Omega \mathrm{m}$, também podem conter aqüíferos de interesse secundário para exploração.

Este aqüífero principal situa-se a uma profundidade média entre $80 \mathrm{~m}$ e $100 \mathrm{~m}$. Com base em dados de poços perfurados no centro da àrea inferimos que sua espessura não é inferior a 70m. Já na região litorânea ou seja no sopé das falésias da Formação Barreiras, a profundidade do topo do aquífero diminui para cerca de $60 \mathrm{~m}$. Os dados de poços indicam a presença de um arenito produtor nesta profundidade. Supondo que se trata da mesma 


\begin{tabular}{|l||c||c||c||c||c||c|}
\hline \multicolumn{7}{|c|}{ Curva do tipo Q } \\
\hline Modelo & $\rho_{1}$ & $\rho_{2}$ & $\rho_{3}$ & $h_{1}$ & $h_{2}$ & RMS(\%) \\
\hline Verdadeiro & 200,00 & 100,00 & 20,00 & 10,00 & 200,00 & \\
\hline Inicial & 230,00 & 85,00 & 35,00 & 7,50 & 250,00 & 27,67 \\
\hline Invertido $L^{1}$ & 199,99 & 100,00 & 19,99 & 9,99 & 199,99 & 0,05 \\
\hline Invertido $L^{2}$ & 202,44 & 102,73 & 18,85 & 9,94 & 204,58 & 3,20 \\
\hline Invertido ILRMD & 199,99 & 100,00 & 20,00 & 10,00 & 199,99 & 0,003 \\
\hline \multicolumn{7}{|c||}{ Curva do tipo K } \\
\hline Modelo & $\rho_{1}$ & $\rho_{2}$ & $\rho_{3}$ & $h_{1}$ & $h_{2}$ & RMS(\%) \\
\hline Verdadeiro & 40,00 & 800,00 & 10,00 & 10,00 & 60,00 & - \\
\hline Inicial & 50,00 & 650,00 & 6,00 & 7,00 & 50,00 & 39,02 \\
\hline Invertido $L^{1}$ & 39,99 & 768,40 & 9,93 & 9,93 & 62,49 & 2,60 \\
\hline Invertido $L^{2}$ & 39,82 & 812,99 & 11,03 & 9,94 & 57,59 & 4,64 \\
\hline Invertido ILRMD & 39,99 & 800,17 & 9,99 & 10,00 & 59,98 & 0,05 \\
\hline \multicolumn{5}{|c||}{ Curva do tipo H } \\
\hline Modelo & $\rho_{1}$ & $\rho_{2}$ & $\rho_{3}$ & $h_{1}$ & $h_{2}$ & $\mathrm{RMS}(\%)$ \\
\hline Verdadeiro & 100,00 & 20,00 & 800,00 & 15,00 & 50,00 & \\
\hline Inicial & 126,50 & 15,00 & 1000,00 & 12,00 & 40,00 & 25,29 \\
\hline Invertido $L^{1}$ & 99,97 & 18,78 & 790,65 & 15,45 & 46,73 & 4,49 \\
\hline Invertido $L^{2}$ & 99,37 & 21,24 & 1154,96 & 14,93 & 55,56 & 14,69 \\
\hline Invertido ILRMD & 100,03 & 20,00 & 799,68 & 14,99 & 50,02 & 0,04 \\
\hline \multicolumn{5}{|c||}{ Curva do tipo A } \\
\hline Modelo & $\rho_{1}$ & $\rho_{2}$ & $\rho_{3}$ & $h_{1}$ & $h_{2}$ & $\mathrm{RMS}(\%)$ \\
\hline Verdadeiro & 15,00 & 80,00 & 180,00 & 5,00 & 58,00 & \\
\hline Inicial & 19,50 & 60,00 & 234,00 & 3,50 & 34,80 & 16,91 \\
\hline Invertido $L^{1}$ & - & - & - & - & - & - \\
\hline Invertido $L^{2}$ & 15,07 & 83,90 & 190,84 & 4,93 & 71,68 & 9,17 \\
\hline Invertido ILRMD & 14,99 & 80,00 & 180,00 & 4,99 & 58,00 & 0,09 \\
\hline
\end{tabular}

Tabela 2 - Resultados da inversão de SEVs sintéticas utilizando os métodos de IL e ILRMD.

Table 2 - Results from inversion of synthetic VES using the IL and LIMWD.
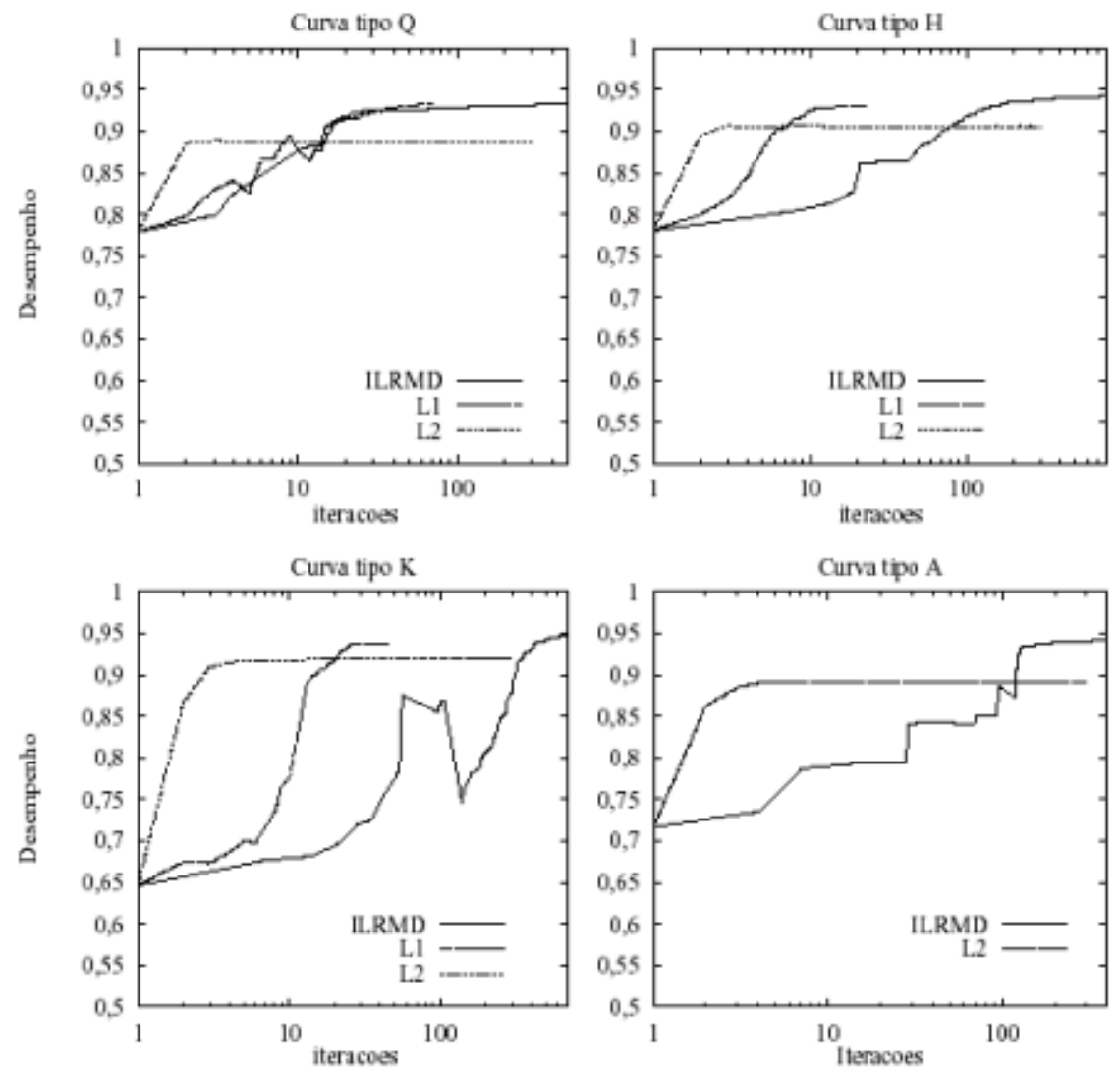

Figura 10 - Curvas de evolução das medidas de qualidade dos ajustes para as inversões das SEVs dos tipos Q, H, K e A, utilizando-se os métodos de IL com normas $\mathrm{L}^{1}, \mathrm{~L}^{2}$ e o método ILRMD.

Figure 10 - Evolution curves of quality measures of adjustments for the VES inversions of $Q, H, K$ and A types by using LI methods with $L^{1}$ and $L^{2}$ norms and the LIMWD method. 
camada aquíffera e sabendo que as falésias da Formação Barreiras se originaram no Quaternário, a partir de erosão marinha (Freitas, 1951; Ghignone, (1979); in Saadi, 2000), é plausível admitir que esta profundidade menor esteja relacionada simplesmente ao desnível topográfico existente entre a Formação Barreiras e os cordões litorâneos.

\section{CONCLUSÕES}

O método de inversão linearizada com reponderação múltipla dos desvios, ILRMD, foi aplicado para a inversão de SEVs da região de Porto Seguro. Este método explora de forma mais extensiva o espaço dos modelos se mostrando eficaz e mais robusto do que os método de IL que utiliza a norma $\mathrm{L}^{2}$ ou L ${ }^{1}$. A estabilidade numérica do método ILRMD utilizado sugere seu emprego a outros problemas de inversão de dados geofísico.

Os resultados da inversão de SEVs da região de Porto Seguro, seguida da interpretação geofísica integrada às informações geológicas disponíveis permitiram delinear o topo do principal aquíf́ero da região. As informações obtidas da geologia de subsuperfície na área foram de grande valia e validam o modelo interpretado. A camada profunda de menor resistividade que as sobrepostas pôde ser confirmada como uma espessa camada de arenito saturado representando o principal aquíf́ero na região. Esse aquiífero ocorre em toda a região a uma profundidade de $80 \mathrm{~m}$ a $100 \mathrm{~m}$ aproximadamente estando recoberto por sedimentos areno-argilosos da Formação Barreiras, com resistividades da ordem de $300 \Omega \mathrm{m} \mathrm{a}$ $500 \Omega \mathrm{m}$. Este horizonte corresponde a um arenito saturado com água doce e representa a opção de captação de água subterrânea mais promissora para o município de Porto Seguro.

Devido aos altos índices pluviométricos nesta região onde não há estação seca durante o ano, é razoável admitir-se que não ocorra problemas de recarga dos aquífferos. Pode-se portanto propor a perfuração de uma série de poços posicionados sobre a Formação Barreiras na parte central da área, que foi identificada como a mais promissora. Tais perfurações devem ser seguidas de perfilagem geofísica com o objetivo de maximizar o desempenho dos poços e o aproveitamento do aqüífero. Os poços poderiam abastecer a população de Porto Seguro, que no auge da alta estação recebe um fluxo turístico enorme. Nesse período a água oriunda do rio dos Mangues não é suficiente, e a água dos poços existentes dentro da cidade, segundo informações obtidas no local junto as empresas de perfuração, contem óxido de ferro acima dos limites de potabilidade.

\section{APÊNDICE}

Neste apêndice apresentamos, na página 290, os passos do algoritmo de inversão baseado no método ILRMD. Também são apresentados exemplos de sua aplicação na inversão de curvas de SEVs dos tipos A, H, K e Q. O algoritmo foi implementado de forma a permitir que a cada iteração um conjunto fixo de 50 modelos candidatos fossem pesquisados, ou seja, 50 valores pre-definidos de $\mathrm{p}$ foram utilizados.

\section{Exemplos de inversão de SEVs sintéticas}

O algoritmo de ILRMD foi testado na inversão de dados sintéticos correspondentes a curvas de SEVs e os resultados foram comparados com aqueles obtidos com a IL conduzida com base nas normas $\mathrm{L}^{1}$ e $\mathrm{L}^{2}$. Para tanto utilizou-se os modelos de curvas padrões de SEVs de três camadas A, H, K e Q, apresentados na Tab. 1. Nestas curvas foi adicionado ruído aleatório em alguns pontos variando entre $5 \% \mathrm{e}$ $15 \%$ para simular desvios que ocorrem em uma situação real. Na Fig. 10 estão representadas as curvas de desempenho que mostram a evolução dos ajustes dos modelos gerados durante o processo de IL. As curvas de desempenho partem do mesmo ponto, correspondente ao mesmo modelo inicial, e ao final do processo observa-se que o maior desempenho é obtido com o método ILRMD. Notase que após um certo número de iterações as curvas de desempenho associadas às normas fixas $\mathrm{L}^{1}$ ou $\mathrm{L}^{2}$ estabilizam num patamar, indicando que o processo de inversão está aprisionado num mínimo local cujo valor de desempenho é menor do que aquele obtido ao final do processo com o método ILRMD, conforme se observa naquelas figuras. Por outro lado, a curva de desempenho associada ao método de ILRMD evidencia a habilidade do método em evitar 


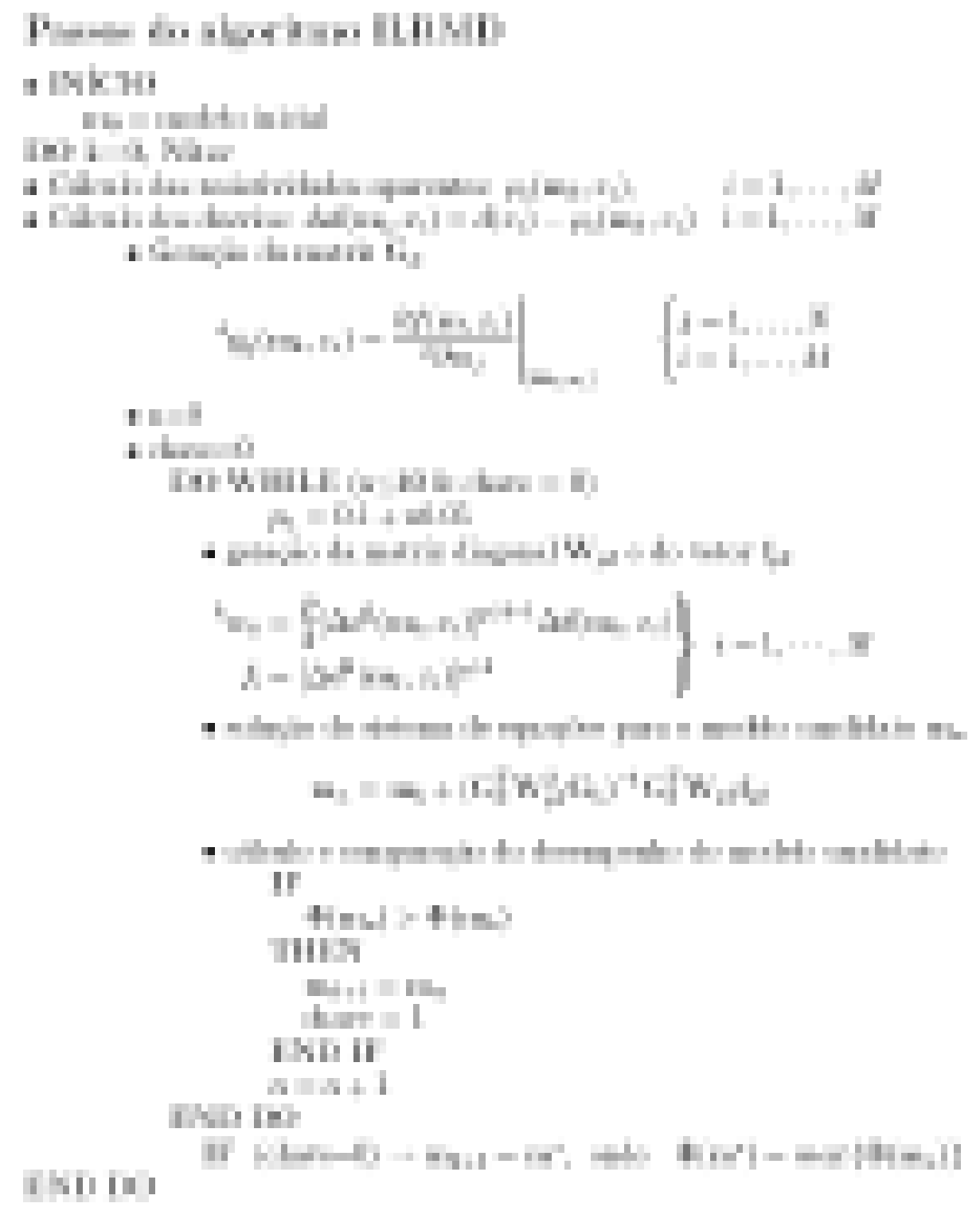

o aprisionamento do modelo corrente em mínimos locais o que não acontece com a IL governada pelas normas $\mathrm{L}^{1} \mathrm{eL}^{2}$. Estas divergiram invarialvelmente ao longo do processo de inversão. A norma $\mathrm{L}^{1}$ divergiu logo ao início da inversão da curva do tipo A. A Tab. 2 mostra os resultados numéricos obtidos para as inversões das resistividades e espessuras para os 4 tipos de SEVs estudados Os pequenos valores do erro médio quadrático (RMS) na Tab.2 demonstram a superioridade do método de ILRMD comparado aos resultados obtidos com IL utilizando normas $\mathrm{L}^{2}$ ou $\mathrm{L}^{1}$.

\section{AGRADECIMENTOS}

Alexandre C. Monteiro agradece à CPRM pela oportunidade de realização do mestrado na UFBA e Milton J. Porsani agradece ao $\mathrm{CNPq}$ pelo apoio à pesquisa. Os autores agradecem ao geólogo Alberto Vieira pelas informações e dados fornecidos e aos revisores anônimos, pelas críticas e sugestões que muito contribuiram para melhorar a apresentação deste trabalho.

\section{REFERÊNCIAS}

Barbosa, J. S. F. \& Dominguez, L. J. M., 1996. Geologia da Bahia: texto explicativo para o mapa geológico ao milionésimo. Salvador: Secretaria da Indústria, Comércio e Mineração. Superintendência de Geologia e Recursos Minerais: 400 pp.

Bittencourt, A. C. S. P., Villas Boas, G. S., Flexor, J. M.\& Martin, L., 1979. Geologia dos depósitos quaternários no litoral do Estado da 
Bahia. In: INDA, H (Ed.), Geologia e Recursos Minerais do Estado da Bahia. Textos Básicos, 1: 2-21, SME/CPM, Salvador

Carrasquilla, A. \& Rijo, L., 1991. Análise da teoria convencional dos meios estratificados na interpretação de SEV's da Ilha do MarajóPará.Rev. Bras. Geofis., 9 (2): 211-222.

Egbert, G. D. \& Booker, J. R., 1986. Robust estimation of geomagnetic transfer function. Geophysics, J. Roy. Astr. Soc. 87: 173-194.

Ferreira, N. R. \& Porsani M. J., 1997. Inversão de sondagem elétrica vertical utilizando algoritmos genéticos combinados com inversão linearizada. V Congresso Internacional da Socidade Brasileira de Geofísica: 646-648.

Gosh, D. P., 1971a. The application of linear filter theory to the direct interpretation of geoelectrical resistivity soundings measurements. Geophysical Prospecting, 19: 192-217.

Gosh, D. P., 1971b. Inverse filter coefficients for the computation of apparent resistivity standard curves for a horizontally stratified earth. Geophysical Prospecting, 19: 769-775.

IBGE, 1987. Folha SE. 24 Rio Doce, geologia, geomorfologia, pedologia, vegetação, uso potencial da terra, Fundação Instituto Brasileiro de Geografia e Estatística, Rio de Janeiro:IBGE, 548pp., 6 mapas:il. -(levantamento de recursos naturais; V. 34)

Johansen, H. K., 1977. A Man/Computer interpretation system for resistivity soundings over a horizontally stratified earth. Geophysical Prospecting 25: 667-691.

Keller, G. V. \& Frischknecht, F. C., 1966. Electrical methods in geophysical prospecting. New York, Pergamon Press.

Koefoed, O., 1979. Geosounding Principles, Elsevier, Amsterdam.

Lines, L. R. \& Treitel, S., 1984. Tutorial - A review of least squares inversion and its application to geophysical problems. Geophysical Prospecting, 32(2): 159-186.
Marinho, J. M. L., 1997. Inversões de sondagens de eletroresistividade com aplicação ao estudo de aquíiferos clásticos na região Acaraú - Itarema, Ceará, Tese de Doutorado, UFBA, Brasil.

Monteiro A. C. \& Porsani M. J., 1999. Inversões de sondagens elétricas verticais da região de Porto Seguro - BA utilizando norma Lp variante: VI Congresso Internacional da Sociedade Brasileira de Geofísica, Resumos Expandidos em CDROM, Rio de Janeiro.

Monteiro, A. C., 1999. Eletrorresistividade aplicada na avaliação do potencial aqüífero da região de Porto Seguro. Dissertação de Mestrado, UFBA, Brasil: 103 pp.

Pelton, W. H., Rijo, L. \& SWIFT, C. M., 1978. Inversion of two-dimensional resistivity and induced-polarization data.Geophysics, 43: 788803.

Porsani, M. J., Stoffa, P. L., Sen, M. K. \& Chunduru, R. K., 2000. Fitness functions, genetic algorithms and hybrid optimization in seismic waveform inversion. Journal of Seismic Exploration, 9: 143-164.

Porsani, M. J., Niwas, S. \& Ferreira, N. R., 2001. A robust inversion of vertical electrical sounding data using a multiple reweighted least-squares method. Geophysical Prospecting, Holanda, 49 (2): 255-264.

Rijo, L., Pelton, W. H., Feitosa, E. C., \& Ward, S. H., 1977. Interpretation of apparent resistivity data from Apodi Valley, Rio Grande do Norte, Brazil. Geophysics, 42: 811-822.

Saadi, A., 2000. Projeto Porto Seguro - Santa Cruz de Cabrália: relatório final. Salvador, CPRM, (1999). cap. 2.

Scales, J., \& Gersztenkorn, A., 1988. Robust methods in inverse theory. Inverse Problem, 4: 1071-1091.

Sen, M., \& Stoffa, P. L., 1995. Global optimization methods in geophysical inversion. Elsevier, Amsterdam: $281 \mathrm{pp}$. 


\section{DEFINITION OF THE AQUIFER TOPINTHE REGION OF PORTOSEGURO-BAHIA FROMTHE INVERSION OF VERTICAL ELECTRICAL SOUNDINGDATA}

This paper comprises geophysical studies carried out with the resistivity method in the Porto Seguro region, in the state of Bahia (Brazil). The objective was to find the main aquifer of that region. Sixty vertical electrical soundings (VES) were performed using the Schlumberger array. The inversion of the VES was done based on the method that uses the linearized inversion with multiple weight for the deviations (LIMWD).

Formulation of the inverse weighted LS problem. Following the procedure proposed by Porsani et al. (2001), we can write the deviations between the observed apparent resistivity and the computed one as,

$$
\begin{gathered}
f(\mathrm{~m}, p, x)=\left\{\left[\Delta d^{2}(\mathrm{~m}, x)\right]^{1 / 2}\right\}^{p}= \\
\left\{\left[\left(d(x)-\rho_{a}(\mathrm{~m}, x)\right)^{2}\right]^{1 / 2}\right\}^{p} .
\end{gathered}
$$

We now quasi-linearize $f(\boldsymbol{m}, p, x) \approx \tilde{f}(\boldsymbol{m}, p, x)$, using a Taylor series expansion on the current model, $\boldsymbol{m}_{k}$ by retaining only the first derivative term. The resulting system of equations can be represented as,

$$
\begin{gathered}
\mathrm{f}(\mathrm{m}, p, \mathrm{x}) \approx \mathrm{f}(\mathrm{m}, p, \mathrm{x})= \\
\mathrm{f}_{p k}-\mathrm{W}_{p k} \mathrm{G}_{k} \Delta \mathrm{m},
\end{gathered}
$$

where $\boldsymbol{G}_{k}$ is the sensitivity matrix, $\Delta \boldsymbol{m}=\boldsymbol{m}-\boldsymbol{m}_{k}$ is the parameter vector correction, $\boldsymbol{f}_{p k}=f\left(\boldsymbol{m}_{k}, p, x\right)$ is the vector of the data deviation whose elements are calculated with equation (8) for $x_{i}, i=1, \ldots, M$, and $\boldsymbol{W}_{p k}$ is a weighting diagonal matrix. By solving the linear system of equation in the $L S$ sense, an expression for updating the current model may be obtained. This approach provides more flexibility to the LS method. During the inversion it allows us to weight the deviation by using a fixed or variable exponent $p$. As illustrated by Fig. 3 at each iteration many candidate models may be obtained.

Inversion of VES data. As shown in Figs. 6 and 2, sixty VES were carried out and inverted. Fig. 8 shows a geoelectrical section in the northern part of the area (A - A'). Fig. 9 shows a map of the top of the deep aquifer. Conclusions. Geoelectrical sections were drawn from the inverted results of the thickness and resistivity of the layers. The interpretation of these sections provided the definition of the main aquifer for ground water exploitation in this region. This aquifer is confined to saturated sandstone layer in the mid-north portion of the area at about $80 \mathrm{~m}$ deep and an average thickness of $70 \mathrm{~m}$. This aquifer represents the best option for ground water exploitation to supply the city of Porto Seguro.

\section{NOTE ABOUT THE AUTHORS}

\section{Alexandre Costa Monteiro}

B. C. em Geologia pela UERJ, 1988. Mestre em Geofísica pela UFBA em 1999. De 1989-1990, geólogo nas Empresas Brunibrás Mineração Ltda, atuando na área de prospecção de granito ornamental. De 1991-1992 geólogo da Empresa Geomecânica S/A., atuando na execução de sondagens geotécnicas rotativas e a percussão. De 1994-2001 atuou como geólogo da CPRM na Divisão de Geofísica no processamento de dados aerogeofísicos. Atualmente está vinculado à PETROBRAS onde atua como geofísico.

\section{Milton José Porsani}

B.C. em Geologia pela USP, 1976. Licenciado em Geologia pela Facudade de Educação da USP, 1977. Mestre em Geofísica pela UFPA, 1981. Doutor em Geofísica pela
UFBA, 1986. Pós-doutorado em Geofísica, Institute for Geophysics at University of Texas at Austin, EUA, setembro/92 a outubro/93. De 1979 a 1982 desenvolveu atividades de pesquisa ligadas aos projetos de prospecção de água subterrânea na Ilha de Marajó e Serra de Carajás. De 1986 até o presente é pesquisador do CPGG/UFBA onde coordena o Programa de Exploração de Petróleo. Em 1990 foi contratado pela UFBA mediante concurso público para professor do Departamento de Geologia e Geofísica Aplicada do IGEO. Desde 2000 é professor Titular na matéria Exploração de Petróleo. Pesquisador do CNPq, nível I-B. Tem atuado no desenvolvimento de métodos e algoritmos de filtragem e processamento de dados sísmicos e na inversão de dados sísmicos e elétricos. 